\title{
Preparation and adsorption property of aminated cross linking microbeads of GMA/EGDMA for bilirubin
}

\author{
ZHIPING CHEN, BAOJIAO GAO* and XIAOFENG YANG \\ Department of Chemical Engineering, North University of China, Taiyuan, 030051, China \\ e-mail: gaobaojiao@126.com
}

MS received 17 December 2008; revised 13 July 2009; accepted 17 July 2009

\begin{abstract}
Cross linking microbeads with a controllable diameter were synthesized by suspension copolymerization of the monomer glycidyl methacrylate (GMA) and the cross linking agent ethylene glycol dimethylacrylate (EGDMA). By the ring-opening reaction of the epoxy groups, the microbeads GMA/EGDMA were modified with different aminating agents and resulting in the aminated microbeads. The morphology of the microbeads was characterized by SEM. The adsorption property of aminated microbeads for bilirubin was investigated, and the effects of various factors, such as the chemical structures of the aminating agents, $\mathrm{pH}$ values of the medium and the presence of bovine serum albumin in the adsorption medium, on the adsorption property were examined. The experimental results show that the aminated microbeads have strong adsorption ability for bilirubin, and the isotherm adsorption behaviour is fitted to Freundlich equation satisfactorily. The adsorption ability of the aminated microbeads modified with hexanediamine is stronger than that of others, and the longer the molecule of multi-ethylene multiamine, the weaker the adsorption ability for bilirubin. The $\mathrm{pH}$ value of the medium affects the adsorption ability greatly, as $\mathrm{pH}=6$, the adsorption ability is strongest. In the presence of BSA, the microbeads still have a higher adsorption capacity towards bilirubin.
\end{abstract}

Keywords. Bilirubin; glycidyl methacrylate; cross linking microbeads; amination; adsorption.

\section{Introduction}

Bilirubin is a principal degradation product of heme catabolisma. The free bilirubin is a lipophilic endotoxin, and under normal physiological conditions, it is transported to liver by albumin for conjugation and subsequent excretion. ${ }^{1,2}$ However, due to a disorder of the liver system in patients, the metabolic pathway of bilirubin is blocked and the amount of unconjugated bilirubin in blood increases. Which interferes with the normal functioning of the cellular machinery and eventually manifests systemic toxicity, ${ }^{1}$ and further may result in fatal kernicterus, ${ }^{3,4}$ and acute renal failure. ${ }^{5}$ An effective treatment for hyperbilirubinemia is hemoperfusion treatment, and a successful hemoperfusion technique requires the absorbent to be of specific, of high adsorption capacity and blood compatible and biocompatible. The preliminary study on the polymer adsorbents had been in progress. The bilirubin molecules contain carboxyl and imine groups (as shown in scheme 1), and the absorbent can adsorb bilirubin via electro-

*For correspondence static interaction or hydrogen bond interaction, so the reported resins used for eliminating bilirubin are usually the polymer containing amine groups (they are usually protonized) and hydroxyl groups. ${ }^{6-8}$ Some researchers also utilized the lipophilic characteristic of bilirubin and adopted polymer immobilized $\beta$ cyclodextrin as adsorbents, because the central hydrophobic cavity of $\beta$-cyclodextrin have an inclusion ability for bilirubin.,

Glycidyl methacrylate (GMA) is a polymer with good biocompatibility, ${ }^{10,11}$ polymer macromoles with epoxy groups are prepared by copolymerization or homopolymerization. When some groups, such as

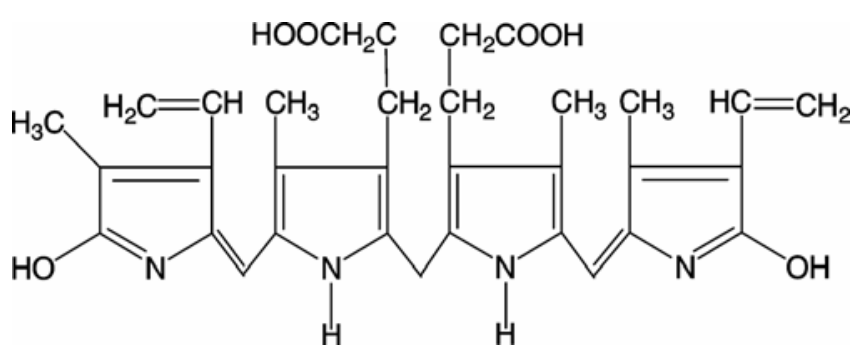

Scheme 1. Chemical structure of bilirubin. 
amine group, hydroxyl group and carboxy group, are drawn in by the ring-opening reaction of epoxy groups, the chemical property modification of polymer is obtained and the polymer can be changed into functional polymer with biocompatibility. ${ }^{12-15}$ In this work, with glycidyl methacrylate (GMA) as monomer and ethylene glycol dimethylacrylate (EGDMA) as cross linking agent, cross linking microbeads with an average diameter of $100 \mu \mathrm{m}$ were synthesized by suspension copolymerization. Subsequently, by the active epoxy groups of GMA, the GMA/EGDMA microbeads were modified with aminating agents of hexanediamine and other three kinds of multiethylene multi-amine. And the adsorption property of the functional microbeads towards bilirubin was studied more in depth. Since there is high density of amine groups on the surface of the functional microbeads, it is expected that the microbeads should have better adsorption property for bilirubin, and the experimented facts confirmed this viewpoint. The designed and prepared biomedical adsorption microbeads in this research not only have excellent adsorption property for bilirubin, but also have biocompatibility.

\section{Experimental}

\subsection{Material and instruments}

Glycidyl methacrylate (GMA) and $\alpha$ - $\alpha^{\prime}$-azoisobisbutyronitrile (AIBN) were obtainted from Fluka, Ethylene glycol dimethylacrylate (EGDMA) was from Yunkai Chemical Limited Company (Yantai City, China), GMA and EGDMA were distilled under reduced pressure before being used. Polyvinyl alcohol (PVA, extent of polymerization 2200) was supplied by Shanwei chemical Limited Company (Hongtong City, China). Hexane diamine (HDA), diethylene triamine (DETA), triethylene tetramine (TETA) and tetraethylene pentaamine (TEPA) were from the chemical reagent factory of Tianjin (Tianjin City, China). Bilirubin (95\% of content) was obtained from Sanshengyuan Biological Science and Technique Limited Company (Chengdu City, China) and Bovine Serum Albumin (BSA) was from Xueman Biological Science and Technology Limited Company (Shanghai City, China).

Used instruments were as follows: 1700 FTIR spectrometer (American Perkin-Elmer Company), DDS-11Ar digit conductivity meter (Leici Instrument Limited Company of Shanghai), Unic-2602
UV spectromenter (American Unic Company), THZ-92 constant temperature shaker equipped with gas bath (Boxun Medical Treatment Equipment Factory of Shanghai), 438VP Scanning Electron Microscope (SEM, England LEO Company).

\subsection{Synthesis and characteristic of cross linking microbeads GMA/EGDMA}

The cross linking microbeads were prepared by suspension polymerization of GMA and EGDMA with AIBN as initiator. The continuous phase, $50 \mathrm{~mL}$ of $\mathrm{NaCl}$ water solution containing PVA, was placed in a four-necked flask equipped with a mechanical stirrer, $\mathrm{N}_{2}$ inlet and a condenser, and when heated to $45^{\circ} \mathrm{C}$, the dispersed phase, $10 \mathrm{~mL}$ of the mixture of GMA and EGDMA, was added. The reaction mixture was stirred for $30 \mathrm{~min}$ and enhanced to $57^{\circ} \mathrm{C}$, then, AIBN was added. The copolymerization was carried out at the constant temperature of $57^{\circ} \mathrm{C}$ for $5 \mathrm{~h}$. The products were filtered off, washed with deionized water and ethanol repeatedly, and dried under vacuum at $50^{\circ} \mathrm{C}$. The chemical structure of the cross linking microbeads GMA/EGDMA was characterized, and their morphology and size (particle diameter) were confirmed by SEM.

\subsection{Preparation and characteristic of aminated cross linking microbeads GMA/EGDMA}

$2 \mathrm{~g}$ of GMA/EGDMA were placed in the mixture of dioxane and deionized water $(1: 1, \mathrm{v} / \mathrm{v})$, made to be sufficiently swelled, and then $\mathrm{NaOH}$ and amine (HAD, DETA, TETA and TEPA ) were added. The reaction was carried out at $80^{\circ} \mathrm{C}$ for $12 \mathrm{~h}$. The products were filtered out, washed repeatedly with distilled water untill the $\mathrm{pH}$ value of the elutant was neutral, dried in a vacuum oven at $50^{\circ} \mathrm{C}$, and the four kinds of aminated microbeads (HDA-GMA/ EGDMA, DETA-GMA/EGDMA, TETA-GMA/ EGDMA and TEPA- GMA/EGDMA) were gained. The amount of amine groups on the surface of the microbeads was determined by $\mathrm{HCl}$-conductometric titration $^{16}$ and the content $(\mathrm{mmol} / \mathrm{g})$ of amine was further calculated.

\subsection{Determining adsorption property of aminated microbeads for bilirubin}

2.4a Determining adsorption isotherm: Under the condition of avoiding light, the aminated micro- 
beads with a fixed amount were added into the bilirubin solutions with different concentrations. The mixtures were shaken on a shaker at a constant temperature of $37^{\circ} \mathrm{C}$ for $80 \mathrm{~min}$ (the time of adsorption equilibrium). Then the supernatant was sucked up and diluted. The absorbance at $438 \mathrm{~nm}$ was determined, and the equilibrium adsorption amounts $Q_{\text {e }}$ $(\mathrm{mg} / \mathrm{g})$ were calculated by (1)

$$
Q_{\mathrm{e}}=\frac{\left(C_{0}-C e\right) V}{1000 m},
$$

where $C_{0}$ and $C_{\mathrm{e}}(\mathrm{mg} / \mathrm{L})$ are the initial and final equilibrium concentrations of bilirubin, respectively, $V(\mathrm{~mL})$ is the volume of the adsorption solution and $m(\mathrm{~g})$ is the mass of the aminated microbeads.

2.4b Examining effects of various factors on adsorption capacity of aminated microbeads: With one of factors varied in series and others fixed, the isotherm adsorption experiments were carried out, so that the effect of some factors, such as the structure of aminating agent, $\mathrm{pH}$ values of the medium and the presence of BSA in the adsorption medium, on the adsorption capacity of the aminated microbeads for bilirubin was researched.

\section{Results and discussion}

\subsection{Preparation of aminated cross linking microbeads GMA/EGDMA}

Due to slightly solubility of GMA in water, suspension copolymerization methods were performed to preparing cross linking microbeads GMA/EGDMA. Meanwhile, in order to decrease the solubility of GMA in water further, an amount of $\mathrm{NaCl}$ was added into the continuous phase to ensure the success of suspension copolymerization. Under the initiation of AIBN, in the suspension droplet the cross linking polymerization of the monomer GMA and EGDMA were happened, and the cross linking microbeads were obtained. On the surface of the cross linking microbeads, a lot of epoxy groups is existed. So, by ring-opening reaction of epoxy groups, the microbeads GMA/EGDMA can be chemically modified easily with aminating agent, such as hexanediamine and multi-ethylene multiamine. And the aminated cross linking microbeads can obtained. The process of synthesization of the aminated cross linking microbeads GMA/EGDMA is demonstrated in scheme 2 .

\subsection{The SEM of cross linking microbeads GMA/EGDMA}

Figure 1 shows the SEM of microbeads GMA/ EGDMA. It can be clearly seen that there is good sphericity, and particle diameter is also homogeneous. The microbeads GMA/EGDMA with different particle diameter were obtained by changing the condition of copolymerization. In this paper, the cross linking microbeads GMA/EGDMA with an average diameter of $150 \mu \mathrm{m}$ were synthesized and

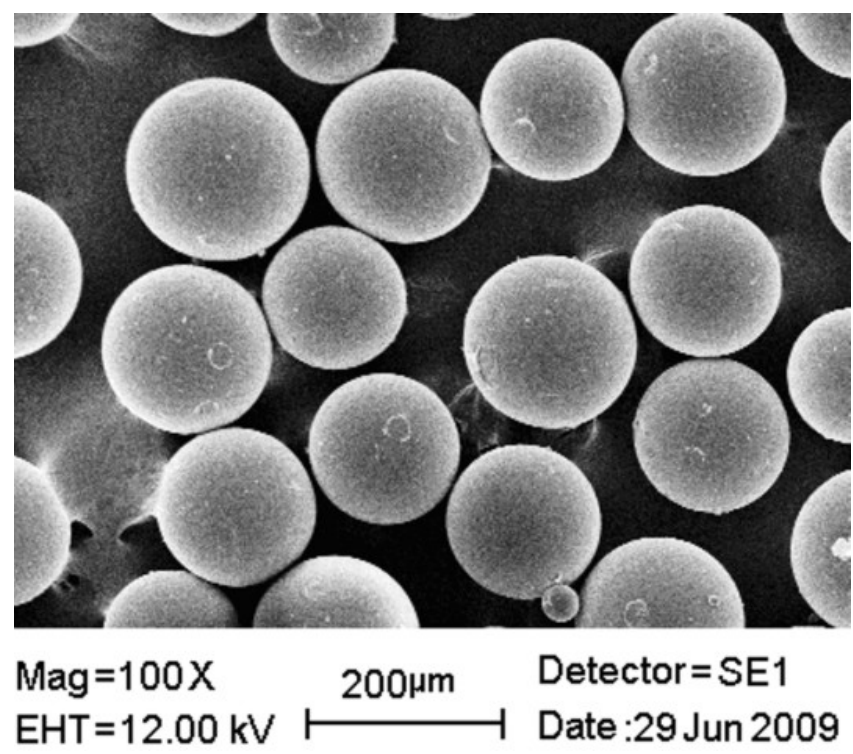

Figure 1. Microscope image of microbeads GMA/ EGDMA

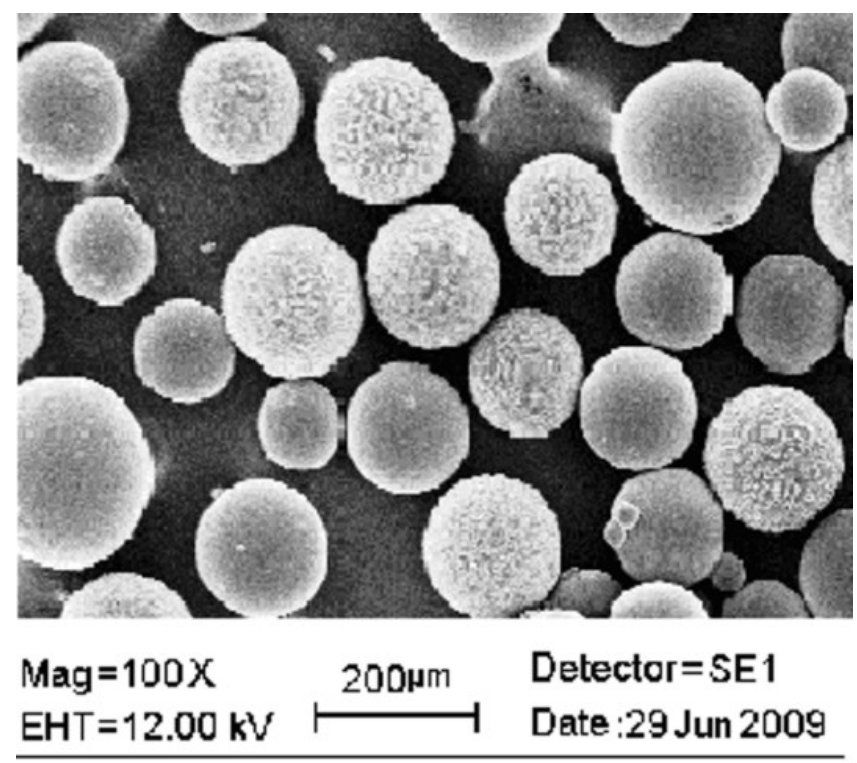

Figure 2. Microscopic image of aminated microbeads HDA-GMA/EGDMA. 
(1) Suspension copolymerization of GMA and EGDMA

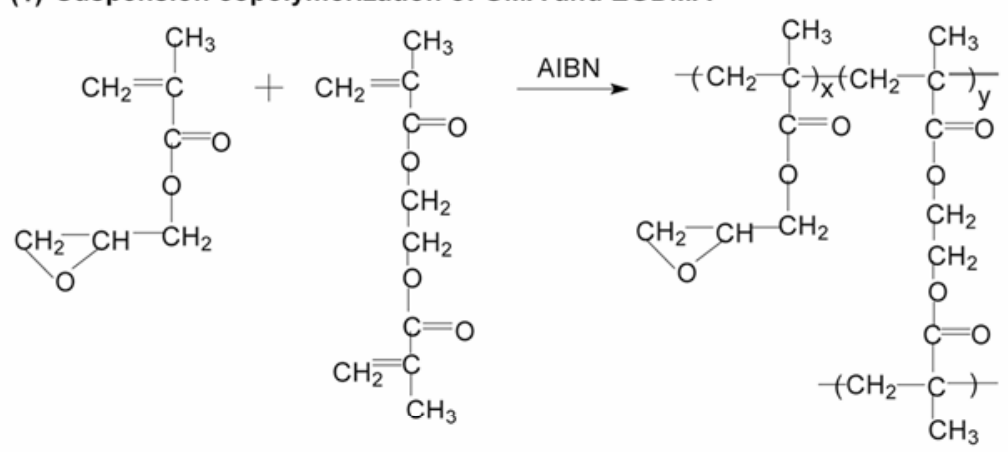

(2) Amination reaction of GMA/EGDMA cross linking microbead with aminating agent

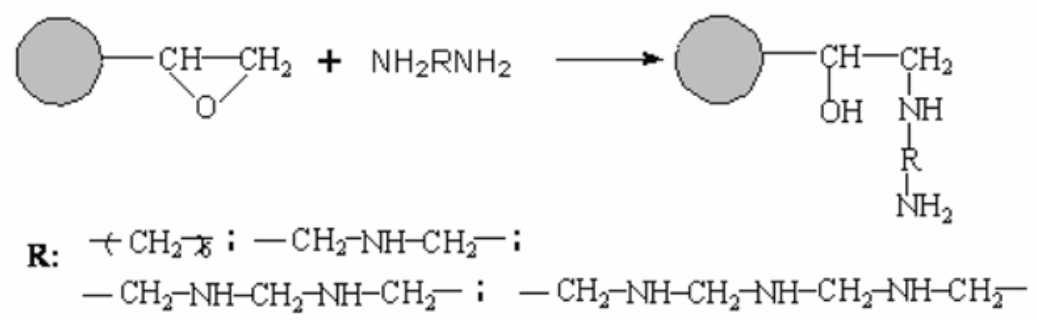

Scheme 2. Schematically expressing the preparation process of aminated crosslinking microbeads of GMA/EGDMA.

used to prepare aminated microbeads. And the SEM of aminated microbeads HDA-GMA/EGDMA was shown in figure 2 . The sphericity of aminated microbeads HDA-GMA/EGDMA is still good. However, the surface of aminated microbeads becomes rough. This shows that ring-opening reaction of epoxy groups on the surface of the microbeads had happened, and aminated microbeads had prepared successfully.

\subsection{Adsorption property of aminated microbeads GMA/EGDMA for bilirubin}

3.3a Adsorption isotherm: The relationship curve between the equilibrium adsorption amount and the equilibrium concentration of four kinds of aminated microbeads GMA/EGDMA for bilirubin at $37^{\circ} \mathrm{C}$ is given in figure 3 . It can be observed that the equilibrium adsorption amount increases with the increasing of the equilibrium concentration of bilirubin. When using the aminated microbeads HDA-GMA/ EGDMA, with $500 \mathrm{mg} / \mathrm{L}$ of the initial concentration of bilirubin, finally the equilibrium concentration changes into $162 \mathrm{mg} / \mathrm{L}$, and the equilibrium adsorption amount gets up to $17.80 \mathrm{mg} / \mathrm{g}$ (more than that in report, ${ }^{17} 0.24 \mathrm{mg} / \mathrm{g}$ ), as shown in figure 3 . The higher adsorption amount suggests that there is a strong affinity interaction between the adsorbent and adsorbate. On the surface of aminated microbeads, there is a lot of amino-group, in water amino-group are protonized and with positive electricity, whereas there are two carboxyls in bilirubin molecule, that can dissociate and with negative electricity, so a strong electrostatic interaction are existed between aminated microbeads and bilirubin. In addition, there are hydrogen-bond interaction between undissociateed carboxyl group and imino of bilirubin and unprotonized amino-group of microbeads. As a result of two kinds of interaction, aminated microbeads can produce a strong adsorption action against bilirubin. Freundlich isotherm and its logarithm form are represented in (2) and (3), respectively.

$$
\begin{aligned}
& Q=k C^{n}, \\
& \lg Q=n \lg C=\lg k .
\end{aligned}
$$

When the data in figure 3 are treated with linear regression according to the logarithm form of Freundlich equation, four straight lines are obtained with regression coefficient in 0.9923, 0.9851, 0.9815 and 0.9899 respectively, as shown in figure 4. These facts indicate that the adsorption of 
bilirubin on the surface of the aminated microbeads fits the Freundlich isotherm, and implies a monolayer adsorption.

\section{3b Effects of sphere size on adsorption property:} Figure 5 shows isothermal adsorption curves of aminated microbeads HDA-GMA/EGDMA at $37^{\circ} \mathrm{C}$ with sphere size $74 \mu \mathrm{m}, 148 \mu \mathrm{m}, 192 \mu \mathrm{m}$, respectively. It can be observed that the equilibrium adsorption amount increases with the decreasing of sphere size of the aminated microbeads at the same equilibrium concentration of bilirubin. The specific surface area of aminated microbeads increases with

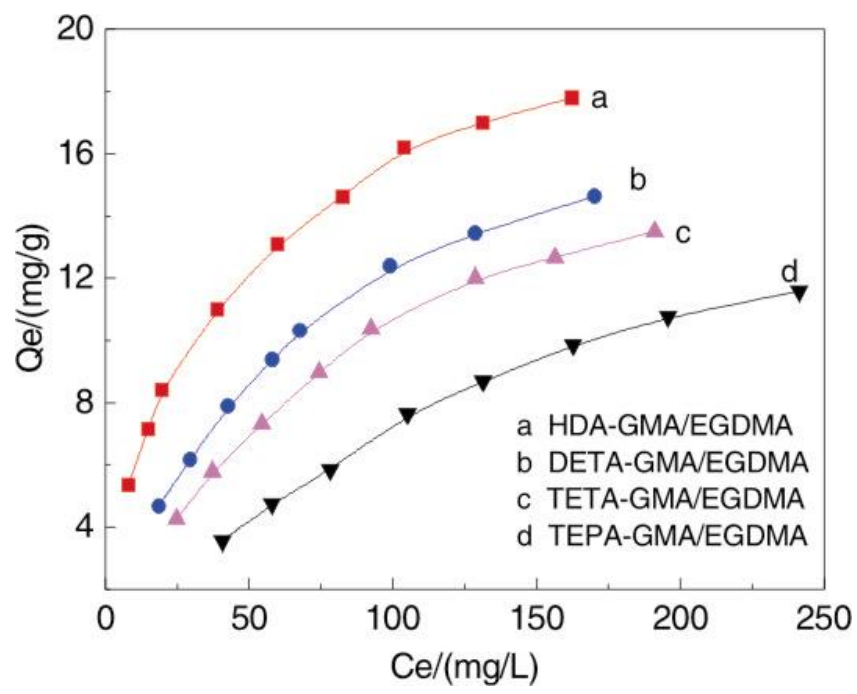

Figure 3. Adsorption isotherm of various aminated microsbeads for bilirubin. Temperature: $37^{\circ} \mathrm{C} ; \mathrm{pH}=7 \cdot 4$.

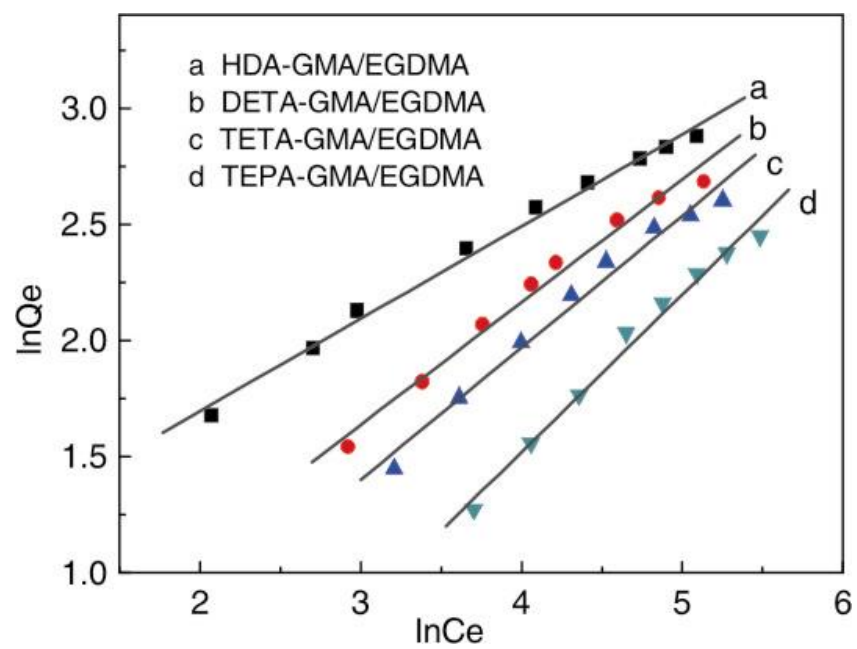

Figure 4. Logarithm relationship between adsorption capacity and equilibrium concentration of bilirubin temperature: $37^{\circ} \mathrm{C} ; \mathrm{pH}=7 \cdot 4$. decreasing of sphere size, which resulted in the number of amino-group on the surface of microbeads adding at the same quality of microbeads. Therefore, the smaller the sphere size, the stronger the adsorption capacity. This fact suggests a monolayer adsorption again.

3.3c Effects of structure of aminating agents on adsorption property: The amount of amine groups on the surface of four kinds of aminated microbeads, HDA-GMA/EGDMA, DETA-GMA/EGDMA, TETAGMA/EGDMA and TEPA-GMA/EGDMA, were $4.45,4.36,4.34$ and $4.00 \mathrm{mmol} / \mathrm{g}$ respectively. So, the difference of the amount of amine groups on these microbeads is not obvious. In figure 3, two results can be clearly seen: first, the adsorption ability of the microbeads HDA-GMA/EGDMA is superior to that of others; second, the longer the molecule of the multi-ethylene multi-amine, the weaker the adsorption ability for bilirubin. Just as mentioned above, the difference of the amount of amine groups on the surface of microbeads is small, but the change of the adsorption ability for bilirubin is obvious, why? The authors think the reasons is probably the difference of hydrophobic interaction and the difference of steric barrier caused by the varying of connecting arm in length. Bilirubin molecule is lipophilic to a certain extent, that is to say, it is hydrophobic, so the hydrophobic interaction is existed between bilirubin molecule and microbeads substrate GMA/EGDMA, and this kind of interaction is also influenced by the structure of aminating agents. In the microbead HDA-GMA/EGDMA, there

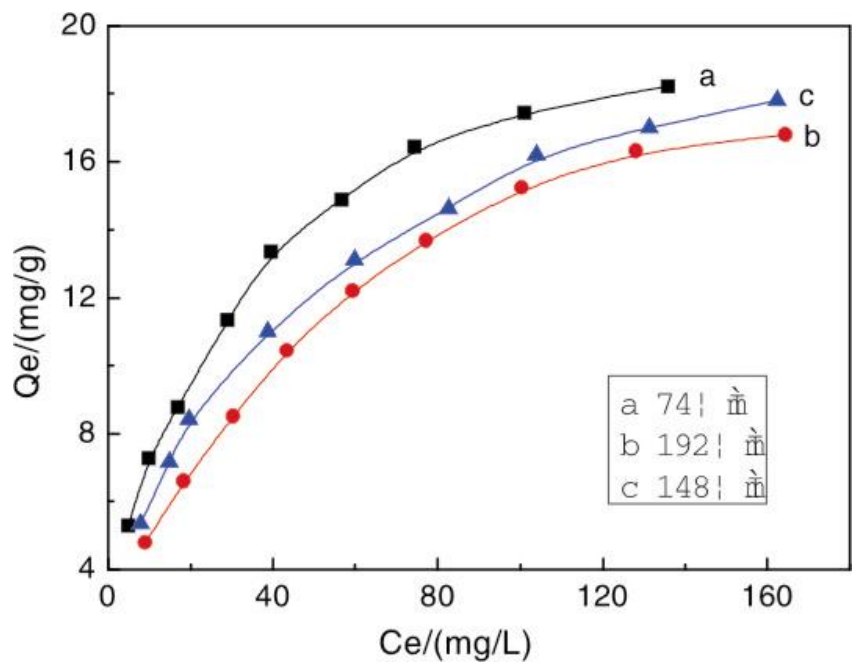

Figure 5. Effect of sphere size on adsorption property of HDA-GMA/EGDMA. Temperature: $37^{\circ} \mathrm{C}$; $\mathrm{pH}=7 \cdot 4$. 
are hydrophobic carbon chains with six carbon atoms, so the hydrophobic interaction, between HDAGMA/EGDMA and bilirubin, is strengthened. This is one of main reasons leading to strong adsorption ability of HDA-GMA/EGDMA microbeads. In addition, for amino end groups of HDA-GMA/EGDMA, there is a spacer with six carbons between it and bilirubin. Obviously, the steric barrier becomes small when bilirubin acts with amino end groups, and this is another reason leading to strong adsorption ability of HDA-GMA/EGDMA microbeads. For the microbeads modified with multi-ethylene multiamine, on one hand, the hydrophobic interaction between microbeads and bilirubin is not reinforced because of hydrophilic of multi-ethylene multiamine molecule; on the other hand, the existence of multi-amine groups maybe lead to the seriousness of additional cross linking and this makes the distance from each amine groups to microbeads substrate to be short. Therefore, there is a bigger steric barrier when bilirubin combines with these amine groups, as a result, the adsorption ability is weaker. The longer the molecule of multi-ethylene multi-amine (such as TEPA), the stronger the action of two kinds negativity influences, just as mentioned above, and the weaker the adsorption ability of bilirubin.

3.3d Effects of $p H$ value on adsorption property: With $100 \mathrm{mg} / \mathrm{L}$ of the initial concentration of bilirubin solution, the adsorption isotherm of aminated microbeads HDA-GMA/EGDMA, DETAGMA/EGDMA and TETA-GMA/EGDMA for bilirubin were produced at different $\mathrm{pH}$ values, and figure 6 displays the relationship curve between the equilibrium adsorption amount and $\mathrm{pH}$ values.

It can be seen from figure 6 , that the effect of $\mathrm{pH}$ values on the adsorption capacities of the microbeads for bilirubin is remarkable. As the $\mathrm{pH}$ values of the medium are lower than 6 , the adsorption capacity is small; the adsorption capacities increase rapidly with the increase of $\mathrm{pH}$ values; as $\mathrm{pH}$ reaches to 6 , the adsorption capacity is the highest; afterwards, the adsorption capacity decreases with the increase of $\mathrm{pH}$ values. The change of the adsorption capacities in different $\mathrm{pH}$ ranges reflect the variation of the electrostatic interaction between the microbeads and bilirubin. The protonized degree of aminated microbeads is strongly affected by $\mathrm{pH}$ value, and the lower $\mathrm{pH}$ value, the more protonized degree. Furthermore, in bilirubin molecule, the dissociation of two propionyloxys strongly depends on the $\mathrm{pH}$ of the solution. So in different $\mathrm{pH}$ ranges, the existing forms of both the amine groups on the surface of microbeads and propionyloxys of bilirubin are different, resulting in the variation of the electrostatic interaction between them. In acidic solution, amine groups on the surface of microbeads has greater cationic degree, whereas the dissociation of the propionyloxy of bilirubin is inhibitied seriously and exist mainly as the form of carboxy group, so the electrostatic attraction between microbeads and bilirubin is very weak, which make the adsorption capacity to be very low. Along with the increase of $\mathrm{pH}$, the dissociation degree of propionyloxys increases and the electronegativity of bilirubin molecules are strengthened, so the electrostatic attraction between microbeads and bilirubin is reinforced and resulting in the increase of the adsorption capacity. According ionization equilibrium constant of propionyloxy $(\mathrm{p} K=4 \cdot 2-4 \cdot 5)^{18}$, when $\mathrm{pH}$ reaches to 6 , the number of ionized propionyloxy is 40 times that of non-ionizing propionyloxy, that is to say, the propionyloxy of bilirubin exists mainly as the negative ion form of carboxy group, and the protonized degree of aminated microbeads still is higher, so the electrostatic attraction between microbeads and bilirubin is very strong. On the other hand, along with the increase of $\mathrm{pH}$, the cationic degree of amine groups on the surface of microbeads decrease, which is not in favour of adsorption for bilirubin, when the $\mathrm{pH}$ values are less than 6 , the negativity influence does not manifest still. However, when $\mathrm{pH}>6$, the cati-

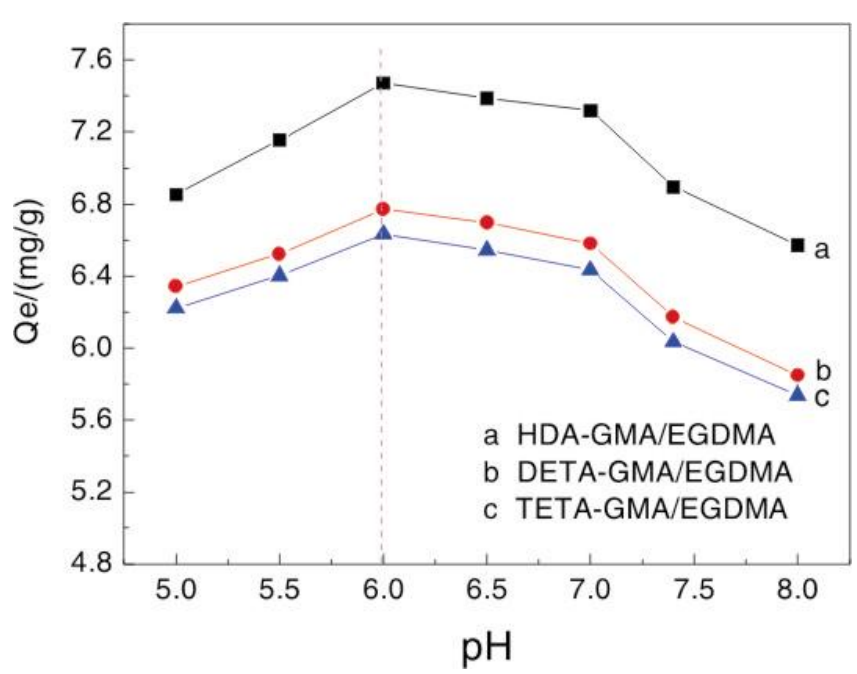

Figure 6. Effect of $\mathrm{pH}$ on adsorption property of aminated microbeads. Temperature: $37^{\circ} \mathrm{C}$; Concentration of bilirubin: $100 \mathrm{mg} / \mathrm{L}$. 
onic degree of microbeads decreases further and the negativity influence manifests, these lead to the less electrostatic interaction of bilirubin and resulting in the decrease of the adsorption capacity. Therefore, as $\mathrm{pH}$ reaches to 6 , the adsorption capacity reaches high.

3.3e Effects of BSA in the adsorption medium on adsorption property: Serum albumin is a natural carrier for bilirubin in the blood depending on the combination of amino-acid residue to bilirubin. Bilirubin in blood exists partially as complex with serum albumin. So, in this paper, the effect of BSA on the adsorption property of HDA-GMA/EGDMA for bilirubin was studied. When the molar ratio of bilirubin to $\mathrm{BSA}$ in the adsorption medium was adopted as $2: 1$, the varying curve of the adsorption capacity with the initial concentration of bilirubin is shown in figure 7(a). It is obvious that the adsorption capacity of the aminated microbeads HDAGMA/EGDMA for bilirubin decreases to a certain extent because of the presence of BSA in the medium. This is the result of complete combination of BSA with the microbeads for bilirubin. However, the microbeads still have a strong adsorption for bilirubin in the presence of BSA. Thus, the aminated microbeads have higher affinity towards bilirubin, and are valuable for the removal of bilirubin in hemoperfusion treatment.

\section{Conclusions}

In this paper, cross linking microbeads GMA/ EGDMA, with an average diameter of $150 \mu \mathrm{m}$ and

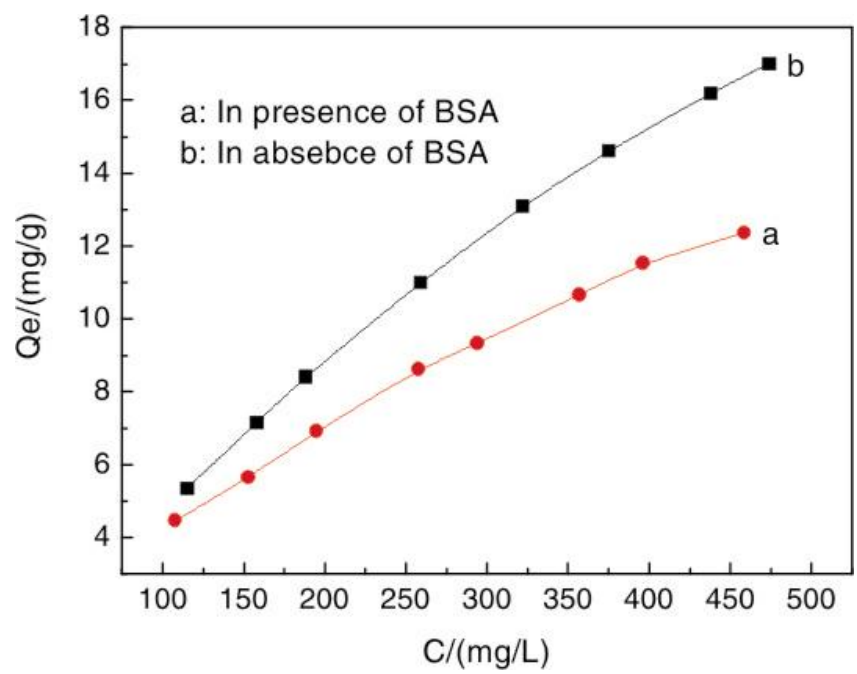

Figure 7. Effect of BSA on adsorption property of aminated microbeads. Temperature: $37^{\circ} \mathrm{C} ; \mathrm{pH}=7 \cdot 4$. good sphericity, were synthesized by suspension copolymerization. Subsequently, by right of the ringopening reaction between amino and epoxy groups, different aminating agents were grafted on the surface of the microbeads, therefore, the different aminated cross linking microbeads were successfully prepared. Since stronger electrostatic interaction between the protonized amine groups on the surface of microbeads and the carboxyls of bilirubin, aminated microbeads GMA/EGDMA exhibit strong adsorption ability against bilirubin. The isotherm adsorption for bilirubin fits the Freundlich equation, and implies a monolayer adsorption. Besides electrostatic interaction, there are hydrogen-bond interaction and hydrophobic interaction between aminated microbeads and bilirubin molecule. The adsorption property of microbeads for bilirubin was affected by the chemical structures of the aminating agents and $\mathrm{pH}$ values of the medium. As in the nearly neutral solutions with $\mathrm{pH} 6$, electrostatic interaction is strongest, and the microbeads have the highest adsorption capacity for bilirubin. The adsorption ability of the aminated microbeads modified with hexanediamine (HDA-GMA/EGDMA) is stronger than that of other aminated microbeads, moreover, the smaller the sphere size, the greater the adsorption properties. In the presence of BSA, the microbeads HDA-GMA/EGDMA still have a higher adsorption capacity towards bilirubin.

\section{References}

1. Ahmad N, Arif K, Faisal S M, Neyaz M K, Tayyab S and Owais M 2006 Biochimica et Biophysica Acta $(B B A)$ - General Subject 1760227

2. Vanstapel F, Blankaert N, Tavaloni N and Berk P D 1993 Hepatic transport and bile secretion, physiology and pathophysiology (New York: Raven Press)

3. Gourley G R 1997 Adv. Paediatr. 44173

4. Hansen T W and Bratlid R D 1986 Acta Pediatr. Scand. 75513

5. Yuan Z, Wei B, Hang D H, Shi L Q, Song Z J and He B L 2000 Chemical Journal of Chinese University 21 731

6. Xia B L, Zhang G L and Zhang F B $2003 \mathrm{~J}$. Membrane Sci. 2269

7. Lu L, Yuan Z, Shi K Y, He B L, Liu B, Shen B and Wang Q S 2003 Chem. J. Chinese University 24454

8. Yu Y H and He B L 1996 Reactive and Functional Polymer 31195

9. Wang H J, Ma J B, Zhang Y H and He B L 1997 Reactive and Functional Polymer 321

10. Amara M and Kerdjoudj H 2003 Desalination 15579

11. Andersson M M and Hatti-Kaul R 1999 J. Biotechnol. 7221 
12. Gao S Y and Yu L G 2003 Acta Polymerica Sinica 34 18

13. Vakurov A, Simpson C E, Daly C L, Gibson T D and Millner P A 2004 Biosensors and Bioelectronics 20 1118

14. Pessela B C C, Fernández-Lafuente R, Fuentes M, Vián A, García J L, Carrascosa A V, Mateo C and Guisán J M 2003 Enzyme and Microbial Technol. 32 369
15. Unsal E, Bahar T, Tuncel $\mathrm{M}$ and Tuncel A $2000 \mathrm{~J}$. Chromatogr. A898 167

16. Jiang P F, Gao B J and Lei H B 2006 Acta Chimica Sinica 64817

17. Syu M J, Deng J H and Nian Y M 2004 Analytica Chimica Acta 504167

18. Gülay B, Yalçın E and Arıca M Y 2005 Colloids and Surfaces A: Physicochemical Engineering Aspects 264195 